\title{
A Global Atmospheric Contaminant Transport Model Based on 3D Advection-Diffusion Equation
}

\author{
Ting Zhang, Ning Xu, Longfei Guo, Yi Huang, and Yong Bai
}

\begin{abstract}
To study the global air pollution concentration and evaluate the impact of regional pollution on whole earth, a global atmospheric contaminant transport model is established based on 3D advection-diffusion equation, which captures the processes of advection by wind and turbulent diffusion during atmospheric dispersion. The Eulerian finite difference method is employed for numerical solution of the proposed model. Then one-year aerosol transport on global scale is simulated using the proposed model, and the simulation results are verified against the real remote sensing data of NASA. It turns out that the simulated values of aerosol concentration are strongly correlated with the real values and the correlation coefficient is above 0.8 for sample point. Furthermore, the proposed model is used for predicting the next-year global atmospheric contaminant concentration by simulation.
\end{abstract}

Index Terms-Global contaminant transport model, advection-diffusion equation, aerosol.

\section{INTRODUCTION}

With the development of economy and the improvement of people's living standard, environmental problems have aroused wide attention. Air pollution is one of the most important concerns and may cause many accompanying problems. Actually, atmospheric acidification, ozone hole, desertification of land, and global warming caused by gases are four main problems faced by human being in the $21 \mathrm{st}$ century[1]-[3]. Since air pollution directly affects the health of people, how to simulate and predict the atmospheric contaminant transport process effectively is an important topic of atmospheric research.

Most of related works on the research topic of atmospheric contaminant transport are on the regional scale [4]-[6]. Those models can simulate the transport process of pollutants by combining with regional climate, terrain, etc. However, such regional models ignore the complex global factors and are not suitable for solving the large-scale intercontinental contaminant transportation problem. Though there are a few of global contaminant transport models for studying the contaminant transport in Porous Media or marine pollutant transmission, most of such

Manuscript received April 5, 2013; revised July 2, 2013. This paper was supported by the National Natural Science Foundation of China under Grant No. 61062006.

Ting Zhang is with the Department of Ocean, Hainan University, 58 Renmin Ave, Haikou, Hainan 570228, China (e-mail: zhangwentingmm@gmail.com).

Ning $\mathrm{Xu}$, Longfei Guo, Yi Huang, and Yong Bai are with College of Information Science \& Technology, Hainan University, 58 Renmin Ave., Haikou, Hainan 570228, China (e-mail: bai@hainu.edu.cn). models are two-dimensional[7]-[9].

Compared with the related models on atmospheric contaminant transport, we propose a model in this paper with three appealing features: three-dimensional (3D), global scale, and targeted for aerosol transport simulation.

In this paper, we present the global 3D atmospheric contaminant transport model and simulate the transport of aerosol around the world. This proposed model uses 3D space coordinates which can fully address the spread of pollutants in space. In this mode, the wind speed and turbulent diffusivity are considered. Furthermore, a comprehensive term is introduced into the model to take into account the factors of emission source, chemical conversion, dry deposition and wet scavenging. The parameters in the model are variables changing over time and space. For simulation purposes, the horizontal resolution of the model is $4^{\circ} \times 5^{\circ}$, and the vertical direction is divided into 11 layers.

We first simulate the aerosol diffusion in the world in one year. It turns out that the simulated global aerosol concentration distribution is consistent with the real aerosol concentration distribution. Actually, the simulated value has strong correlation with the real data, and the correlation coefficient is above 0.8 for sample point. We also predict the global aerosol concentration over next year. Hence, the proposed model in this paper can be a useful tool for predicting the global aerosol concentration. If we combine this model with more precise chemical patterns, the accuracy of the model can be improved and the proposed model can be further extended to study other atmospheric contaminants.

The rest of paper is organized as follows. Section II introduces the proposed global atmospheric contaminant transport model based on 3D advection-diffusion equation. Numerical solution of the proposed model is given in Section III. Section IV presents and discusses the simulation results. Finally, conclusions are drawn in Section V.

\section{GLOBAL ATMOSPHERIC CONTAMINANT TRANSPORT MODEL}

\section{A. Established Model Based on 3D Advection-Diffusion Equation}

Atmospheric dispersion refers to the transport of airborne contaminants via two main processes, advection by wind and turbulent diffusion. Therefore, we establish the model of atmospheric contaminant transport based on the advection-diffusion equation. The advection-diffusion equation is a combination of the advection and diffusion equations, and describes physical phenomena where particles, energy, or other physical quantities are transferred inside a 
physical system due to two processes: advection and diffusion. To model global atmospheric contaminant transport, we extend the $2 \mathrm{D}$ advection-diffusion equation in $3 \mathrm{D}[10]$ as

$$
\frac{\partial c}{\partial t}+u_{x} \frac{\partial c}{\partial x}+u_{y} \frac{\partial c}{\partial y}+u_{z} \frac{\partial c}{\partial z}=k_{x} \frac{\partial^{2} c}{\partial x^{2}}+k_{y} \frac{\partial^{2} c}{\partial y^{2}}+k_{z} \frac{\partial^{2} c}{\partial z^{2}}+\lambda c
$$

where $c$ is contaminant concentration; $t$ is time; $x, y, z$ represent the abscissa, ordinate, and height respectively; $u$ is wind speed and $u_{x}, u_{y}, u_{z}$ represent wind speed in three directions of $x, y, z$ respectively; $k$ is turbulent diffusivity and $k_{x}, k_{y}, k_{z}$ represent turbulent diffusivity in three directions of $x, y, z$ respectively; $\lambda$ is a comprehensive term taking into account of the factors of emission source, chemical conversion, dry deposition and wet scavenging.

\section{B. Initial and Boundary Conditions}

Limit conditions are important in Equation (1) of the contaminant transport model. They are decided by actual geographic information and initial contaminant concentration on the boundaries.

There are mainly two approaches to obtain the initial conditions. One is to set the real pollutant concentration as the initial conditions; the other is to set zero concentration as initial conditions. The latter is viewed as ideal circumstance. The expressions of initial conditions of above two cases are

$$
\begin{gathered}
c\left(x, y, z, t_{0}\right)=c_{0}(x, y, z) \quad(x, y, z \in \Omega) \\
c\left(x, y, z, t_{0}\right)=0 \quad(x, y, z \in \Omega)
\end{gathered}
$$

where $C_{0}(x, y, z)$ is the given concentration condition; $\Omega$ is the spatial scope considered.

In the proposed model, we simplify the realistic initial conditions and combine the above two initialization methods. We collect 83 sample points of contaminant concentration and set them as initial concentration. Then we adopt kriging interpolation method to establish concentration distribution of existing pollution halo. The simulated values of pollutant concentration distribution can be used as initial conditions for prediction as long as we can adjust parameters so that the simulated values is similar with observed values.

In general, there are three kinds of boundary conditions of the advection-diffusion equation: Dirichlet condition (the concentration boundary), Neumann condition (the concentration gradient boundary), and Cauchy Condition (the concentration boundary and the concentration gradient boundary specified at the same time) [11].

Considering the calculation efficiency, we choose the ideal boundary condition, i.e., Dirichlet condition. The top boundary is a constant value of pollutant concentration which does not change over time and space. Bottom boundary is a value of pollutant concentration which changes only over space. We assume that other boundaries of concentrations are infinite. In summary, the expressions of boundary conditions are

$\begin{cases}\text { Top Boundary Condition: } & c\left(x, y, z_{0}, t\right)=c_{0} \\ \text { Bottom Boundary Condition: } & c(x, y, 0, t)=c(x, y, z) \\ \text { Left Boundary Condition: } & c(0, y, z, t)=0 \\ \text { Right Boundary Condition: } & c(\infty, y, z, t)=0 \\ \text { Upper Boundary Condition: } & c(x,-\infty, z, t)=0 \\ \text { Lower Boundary Condition: } & c(x, \infty, z, t)=0\end{cases}$

where $z_{0}$ is the height of boundary layer. $c_{0}$ is the constant pollutant concentration. $c(x, y, z)$ is a concentration value changing only over space.

\section{Representation of Parameters}

Wind speed in Equation (1) should be obtained by real observed values that change over time and space. However it is difficult for computers to simulate. Hence, we introduce the distribution function of wind speed with height which is commonly used in meteorology. The atmospheric boundary layer can be divided into surface layer and Ekman layer. In the surface layer, wind velocity obeys logarithmic distribution [12]. Its expression is

$$
u=\frac{u_{*}}{0.4} \ln \frac{z}{z_{0}}
$$

where $u_{*}$ is friction coefficient.

Turbulent diffusivity $k$ represents the turbulent transport capacity which is related to the properties of turbulence fields. The measured results demonstrate that the vertical turbulent diffusivity is relevant to the conditions of underlying surface and meteorological environment. $k_{z}$ is a function of roughness length $z_{0}$, average wind velocity $u$, temperature gradient $\frac{\Delta T}{\Delta z}$ and ground clearance $z$. In practical application, assuming that $k_{z}$ and $k_{y}$ of ground layer are functions of height $z$ and are relevant with wind and wind sheer. In the proposed model, we employ the following exponential function forms of turbulent diffusivity [12].

$$
\begin{gathered}
k_{x}=0 \\
k_{y}(z)=a z^{m} \\
k_{z}(z)=k_{1}\left(\frac{z}{z_{1}}\right)^{n}
\end{gathered}
$$

where $a, m, n, k_{1}, z_{1}$ are constants determined by real physical conditions.

\section{Numerical SOlution OF THE Proposed Model}

A variety of approaches have been used for solving the advection-diffusion equation including

- Analytical approaches: Fourier series, Green's functions, Laplace transforms, Asymptotics.

- Computational approaches: Eulerian Finite Difference, Finite Volume, Spectral.

Analytical solution methods are limited to a few linear differential equation with constant coefficients. To solve the complex 3D equation, we adopt the Eulerian finite difference method instead of using analytical approaches. Eulerian finite difference method is used to obtain the values of discrete 
points [13]. Using Taylor expansion, the explicit difference representation of Equation (1) is

$$
\begin{aligned}
& \frac{C_{i, j, k}^{n+1}-C_{i, j, k}^{n}}{\tau}+u \cos \alpha \frac{C_{i, j, k}^{n+1}-C_{i, j, k}^{n}}{\Delta x}+u \cos \beta \frac{C_{i, j, k}^{n+1}-C_{i, j, k}^{n}}{\Delta y} \\
& +u \cos \gamma \frac{C_{i, j, k}^{n+1}-C_{i, j, k}^{n}}{\Delta z}=k_{0} \frac{C_{i+1, j, k}^{n}-2 C_{i, j, k}^{n}+C_{i-1, j, k}^{n}}{(\Delta x)^{2}} \\
& +a z^{m} \frac{C_{i, j+1, k}^{n}-2 C_{i, j, k}^{n}+C_{i, j-1, k}^{n}}{(\Delta y)^{2}} \\
& +k\left(\frac{z}{z_{1}}\right)^{a} \frac{C_{i, j, k+1}^{n}-2 C_{i, j, k}^{n}+C_{i, j, k-1}^{n}}{(\Delta z)^{2}}+\lambda C_{i, j, k}^{n}
\end{aligned}
$$

where $\tau$ is time step, $\alpha, \beta, \gamma$ are angles of wind directions with respect to $x, y, z$ axes respectively.

When using this method, the time step and spatial steps need to be determined. We divide the studied global space into $3 \mathrm{D}$ units. In the next section, we describe the horizontal resolution of the spatial units used for simulation, and the time step of our model for simulation is one month.

\section{Simulation of Diffusion of Global Aerosol}

\section{A. Simulation Settings}

Firstly, we view the earth as a $2 \mathrm{D}$ map $(900 \times 1600$ pixels $)$ and consider only latitude and longitude. After evenly dividing the map to $20 \times 20$ grid units, we obtain $45 \times 80=3600$ sample points in $2 \mathrm{D}$. Note that such a division corresponds to $4^{\circ} \times 5^{\circ}$ (latitude $\times$ longitude) $2 \mathrm{D}$ spatial resolution. Next, height is taken into account and a 3D model can be established. Because the atmospheric boundary layer has greatest impact on air pollutant transmission, we only consider the transport of air pollutants in the atmospheric boundary layer (about 1000 meters). We split the atmospheric boundary layer to 11 sublayers (100 meters of each layer). In this way, we can observe aerosol concentration at the heights of 11 sublayers of the globe. Finally, there are total of 39600 sample points in 3D space, and we use discrete difference equation to calculate the concentration dynamics in three dimensional space with time. For simulation, we also set the parameters for each sample point including the coefficient of friction, wind velocity, turbulent diffusivity, surface roughness and the comprehensive term. Each of these coefficients can be set as a constant, or as a variable changing over time and space.

\section{B. Simulation Results and Analysis}

From NASA website, we downloaded the monthly data of 83 sample points of global aerosol concentration in a year.

We assume an ideal situation that the coefficients of friction, wind velocity, turbulent diffusivity, surface roughness of each point are constants. The setting of the comprehensive term $\lambda$ is more complicated. When setting the comprehensive term of the 83 sample points, the value of the comprehensive term is adjusted monthly to make the simulated values are consistent with the real observed values. In realistic situation, the comprehensive term can be obtained from the real meteorologic data. In our simulation, the monthly comprehensive terms are assumed to be same as those of last year. Considering the similarities of the comprehensive term in close areas, we can obtain the comprehensive terms of surrounding areas of the 83 sample points by interpolating the values to its surrounding points. Another thing to be pointed out is that we set the background values of the global pollution to be $1 / 12$ of average concentration of the 83 sample points.

After completing the setting of environmental parameters, we set different colors on sample points to represent the pollution levels: concentration $>1.5$ as severe pollution (red); concentration $i>1.0$ (pink) $>0.5$ (dark blue) $>0.1$ (light blue) $>$ 0.025 (green); the rest points are in yellow to represent little pollution.

The global concentration distribution charts can be obtained by using the collected aerosol concentration of 83 sample points with interpolation method. As shown in Fig. 1, the upper chart is the real aerosol concentration distribution of 83 points in the world in January; the lower chart is the simulated isoconcentration map of the aerosol distribution in January. The concentration of 83 sample points in January is set as initial values. The red points represent the 83 pollution sources. After running the advection-diffusion equation, we observe from the change of colors in the transport of pollution that three layers are contaminated after three months. This means pollutants are not only horizontal propagated, but also vertical transmitted.
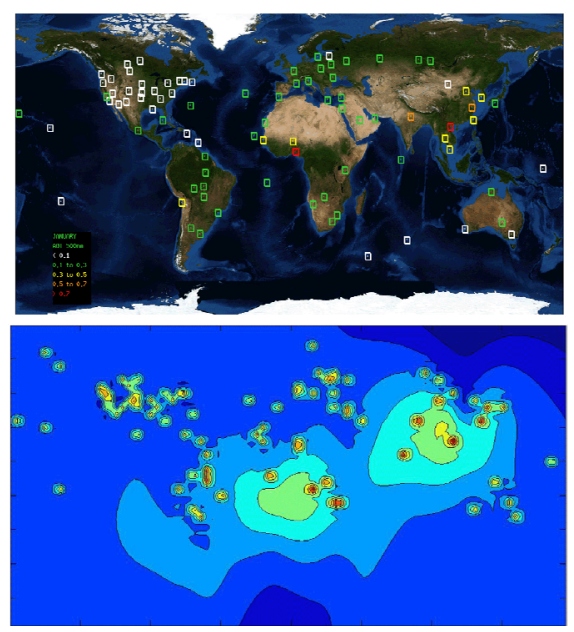

Fig. 1. Real aerosol distribution (upper) and simulated aerosol distribution (lower) in January.
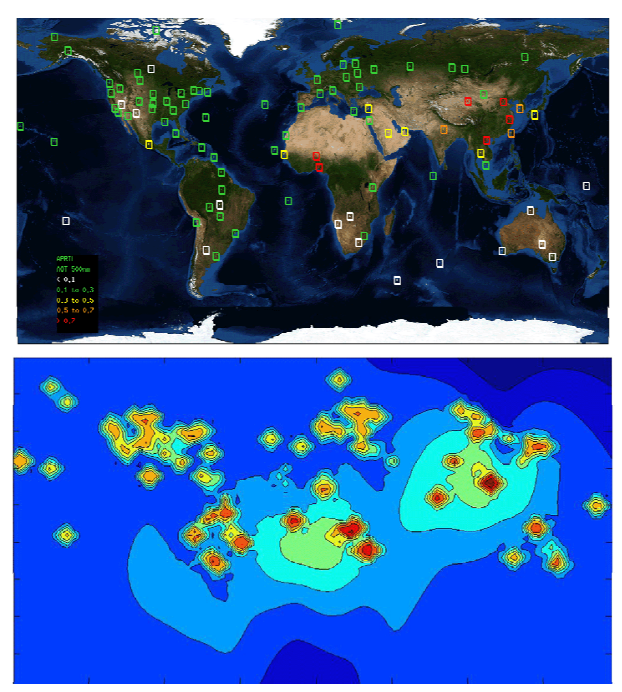

Fig. 2. Real aerosol distribution (upper) and simulated aerosol distribution (lower) in April. 
In Fig. 2, the upper chart is the real aerosol concentration distribution of 83 sample points in April; the lower chart is simulated contamination distribution in April.

By comparing Fig. 1 and Fig. 2, we can see that in January, the color of real aerosol concentration in North America is white $(<0.1)$, which corresponds to the light blue areas in simulated chart. In April, the pollution in North America is worsen and the real values are in green, which corresponds to the orange (medium concentration) regions in simulated chart; In January, the real values of East Asia are in yellow, and the corresponding parts in the simulated chart are in orange (medium concentration); In April, East Asia has more red sample points, and in the simulated chart, the corresponding red (high concentration) areas also increase. Thus, it shows that the model can simulate the global contaminant transport.

We introduce the correlation coefficient to indicate the relevance of real values and simulated values. The calculation formula of correlation coefficient is

$$
\rho_{x y}=\frac{\operatorname{Cov}(X, Y)}{\sqrt{D X} \sqrt{D Y}}
$$

where $X$ is the real value vector, and $Y$ is simulated value vector.

Fig. 3 shows the comparison of the real values and simulated values of aerosol concentration in 12 months in Tomsk. In Tomsk, $\rho_{x y}=0.8323>0.8$, which demonstrates that the simulated values and the real values have strong correlations.

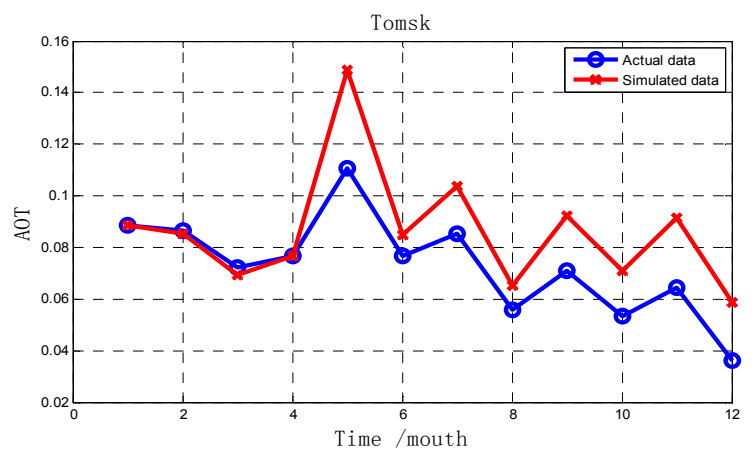

Fig. 3. Real values and simulated values in one year in Tomsk.

\section{Prediction of the Next Year}

In addition, the varying trend of aerosol concentration is also one of the capabilities of our model. Then we use the model to simulate the second year of global aerosol concentration changing trend. Fig. 4 is the simulated aerosol concentration of two years (first year and next year) in Tomsk region.

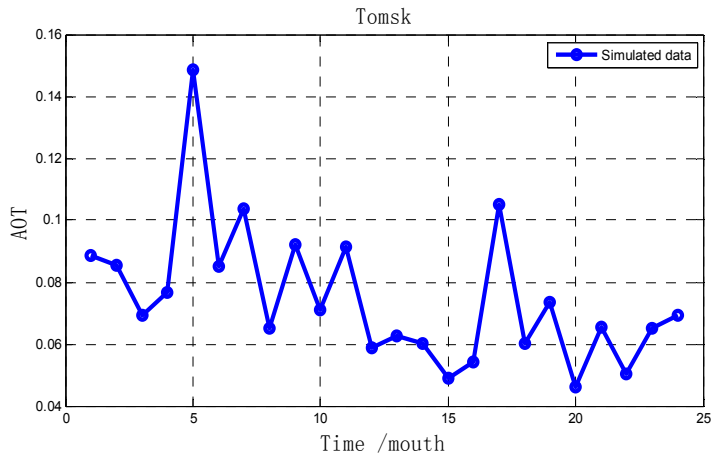

Fig. 4. Simulated aerosol concentration of two years in Tomsk
Actually, the global aerosol diffusion in two years is expanded to more areas. Fig. 5 is the global aerosol concentration distribution chart of the last month in two years. As shown in Fig. 5, the concentration in East Asia is generally high; the trend of the distribution is relatively concentrated in several areas, such as Beijing of China and the Middle East region. Most African countries show a trend of rapid diffusion as well, and this may be caused by the severe emission of industrial pollution in the developing countries.

On the contrast, many developed countries curb industrial pollution and strengthen environmental protection. It can be seen from the map that the diffusion in North America and Western Europe is relatively gentle, and occurs only in limited areas. In addition, the ocean is one of the main sources of aerosols in the world. The sea aerosol concentration and diffusion phenomenon can be observed in the simulated charts, which may be caused by the evaporation of sea salt.

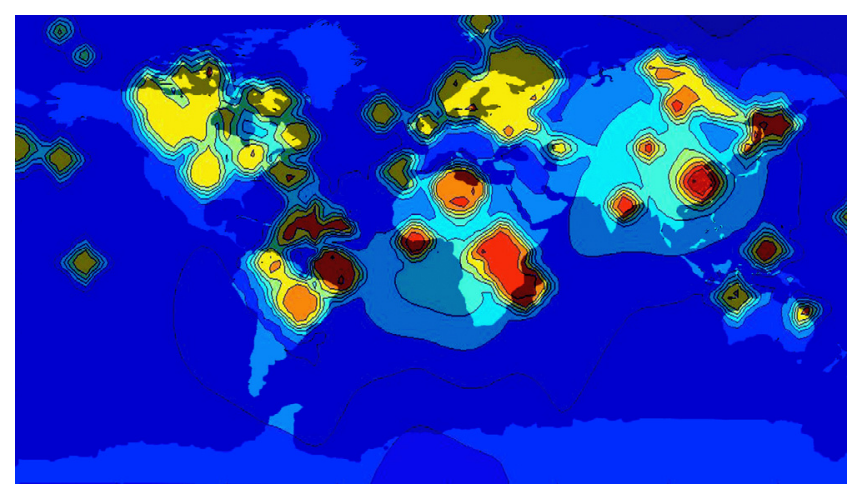

Fig. 5. Global aerosol concentration distribution chart of the last month of two years.

\section{CONCLUSIONS}

In this paper, a global $3 \mathrm{D}$ atmospheric contaminant transport model is established and mainly used for simulating the diffusion of global aerosol. We adopt Euler finite difference method for numerical simulation. In the simulation, the horizontal resolution of our model is $4^{\circ} \times 5^{\circ}$, and the vertical direction is divided into 11 sub-layers. Compared with previous related works, our model can simulate atmospheric contaminant transport on global scale in 3D space. The simulation results of global aerosol are consistent with the observed real remote sensing data of NASA. The proposed model in this paper can be a useful tool for predicting the global aerosol concentration.

At present stage, we only simulate the global aerosol transport with simplified parameterization. If the proposed model takes into account more precise chemical patterns, the accuracy of the model can be improved, and the proposed model can be further extended to study other atmospheric contaminants.

\section{REFERENCES}

[1] J. N. Tester, "Energy and the environment in the 21st century," presented at the Massachusetts Institute of Technology Cambridge, Massachusetts, 1990.

[2] R. W. Corell, "The US Global change research program: An overview and perspectives on TY 1992 program," Bull, AMS, vol. 72, pp. 5.

[3] X. E. Lei, Z.W. Han, and M. G. Zhang, Physical, Chemical, Biological Processes and Mathematical Model on the Air Pollution, China Meteorological Press, 1992. 
[4] J. Hu, "Pollutants Propagation Model Based on Diffusion Equation," Journal of Nantong Vocational College, vol. 26, no. 2, Jun 2012.

[5] Y. P. Hu, "Preliminary study on factors in affecting regional atmospheric environment and prediction method," Journal of Hubei Ecology Vocational College, vol. 4, no. 3, 2006.

[6] J. Wu, "Using regional climate model and atmosphere chemical model to model regional climate and ozone in troposphere over china," Journal of Nanjing University, vol. 38, no. 4, July 2002.

[7] G. H. Huang, "Model contaminant tranport in homogeneous porous media with fractional advection-diffusion equation," Science in China Ser. D Earth Sciences, vol. 48, pp. 295-302, 2005.

[8] H. Wang, "Numerical study and prediction of nuclear contaminant transport from fukushima daiichi nuclear power plant in the North Pacific Ocean," Chinese Science Bulletin, vol. 57, no. 26, Sept. 2012.

[9] P. Q. Chen, "The numerical experiment of the unsteady two dimensional advection-diffusion equation," Atmospheric Physics, Chinese Academy of Sciences, Beijing, vol. 43, no. 1, Feb. 1985.

[10] B. Q. Chen, Numerical Experiments of Unsteady Two Dimensional Advection Diffusion Equation, Acta Meteorologica Sinica, 1985.

[11] C. Y. Li, A Number of Frontal Studies on Atmospheric Science, Hefei: China University of Science and Technology Press, 2009.

[12] W. M. Jiang, Air Pollution Meterorology, Nanjing: Nanjing University Press, 1994, China.

[13] S. Zhang, Finite Difference Numerical Calculation of the Problems for Determining Solution about Parabolic Equations, Beijing: Science Press, 2010, China.

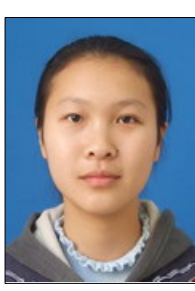

cypris.

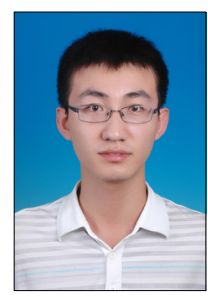

Ning Xu received his B.E. degree from Hainan University, China in 2013, and won the First Class Comprehensive Scholarship of Hainan University in 2010, and the Second Prize of National Mathematical Modeling Competition in 2011, and the First Prize of Central China Mathematical Modeling Competition in 2012. He successfully participated 2013 Interdisciplinary Contest In Modeling. His current research interests include digital image processing.

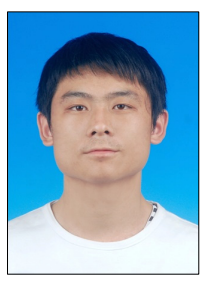

Longfei Guo received his B.S. degree from Hainan University, China in 2013, and won Honorable Mention in American Mathematical Contest in Modeling. His current research interests include economic modeling and risk management.

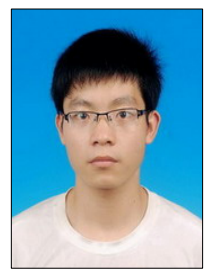

Yi Huang received his B.S. degree from Hainan University, China in 2013, and won the Second Prize in National Mathematical Modeling Competition, Hainan Provincial Division, and First Class Comprehensive Scholarship, Hainan University, and Honorable Mention in American Mathematical Contest in Modeling. His current research interests include systems science and control.

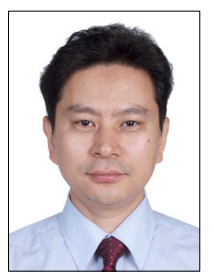

Yong Bai received his B.S. degree from Xidian University, China in 1992, and M.S. degree from Beijing University of Posts and Telecommunications (BUPT), China in 1995, and Ph.D. degree in electrical engineering from Rutgers-The State University of New Jersey, USA in 2001. He was with PacketVideo Corporation, NJ, USA from 2000 to 2002 . He was with Motorola, Beijing, from 2002 to 2004. He was with CEC Wireless from 2004 to 2005 . He was a researcher at DoCoMo Beijing Communication Labs from 2006 to 2009 . He is a professor at College of Information Science \& Technology, Hainan University since 2010. His current research interests include ocean communications and ocean monitoring. 